Pacific Journal of Mathematics

ISOTOPY GALOIS SPACES

Paul Frazier Dual, JR., Peter Fletcher

Vol. 45, No. 2

October 1973 


\section{ISOTOPY GALOIS SPACES}

Paul F. Duvall, Peter Fletcher and Robert A. McCoy

This paper introduces two classes of topological spaces, isotopy Galois spaces and orbit attraction spaces. Both classes of spaces are defined in terms of the actions of the homeomorphism group of a space onto itself, and both classes are closely related to the classes of Galois spaces and representable spaces.

Although there is a metrizable Galois space $X$ such that $X \times X$ is not a Galois space, it is shown that the product of an isotopy Galois space and any Tychonoff space is an isotopy Galois space. Consequently, isotopy Galois spaces include the total spaces of locally trivial fibre spaces with isotopy Galois base and completely regular fibre. From this result it follows that every locally compact topological group which is not totally disconnected is an isotopy Galois space. Since every locally compact totally disconnected topological group is a representable space, every non-discrete locally compact topological group is a Galois space.

We make use of three types of homogeneity which were originally defined and discussed in [3] and [7]. A space $(X, \mathscr{T})$ is strongly 2-homogeneous provided that for any points $x_{1}, x_{2}$ and any points $y_{1}, y_{2}$, there is a homeomorphism $h$ of $X$ onto itself such that $h\left(x_{1}\right)=y_{1}$ and $h\left(x_{2}\right)=y_{2}$. A space $(X, \mathscr{T})$ is nearly homogeneous provided that for any $x \in X$ and any $D \in \mathscr{T}$ there is a $y \in D$ and a homeomorphism $h$ of $X$ onto itself such that $h(x)=y$. A space $(X, \mathscr{T})$ is strongly locally homogeneous if for every neighborhood of any point $x$, there exists a subneighborhood $U(x)$ such that for any $z \in U(x)$ there is a homeomorphism $h$ of $X$ onto itself such that $h(x)=z$ and $h(y)=y$ for each $y \in X-U(x)$. It is evident that every orbit attraction space (defined in $\S 3$ ) is nearly homogeneous and we show that every representable continuum is a strongly 2-homeogeneous orbit attraction space. This result suggests the conjecture that every representable connected space is locally connected. The following two questions concerning homogeneity also remain unresolved.

Question. Is every (metrizable) homogeneous continuum a Galois space?

Question. Is every representable space strongly locally homogeneous? 
Galois spaces and representable spaces are defined and studied in [4], [5] and [6].

All spaces considered in this paper are assumed to be Hausdorff spaces. We also adopt the following notation. Throughout $\boldsymbol{R}$ will denote the real numbers and $S^{1}$ will denote the 1-sphere. If $X$ is a topological space, $H(X)$ will denote the group of homeomorphisms of $X$ onto $X$, and $I d_{X}$ will denote the identity of $H(X)$. If $A \subset X$, then $A^{\prime}=\left\{h \in H(X): h\left|A=I d_{X}\right| A\right\}$. If $G \subset H(X)$ and $A \subset X$, then $G(A)=$ $\{g(a) \mid g \in G, a \in A\}$. We write $x^{\prime}$ instead of $\{x\}^{\prime}$ and $G(x)$ instead of $G(\{x\})$. For the sake of euphony we say on occasion that a homeomorphism $h$ is supported on $U$ to mean that $h \in(X-U)^{\prime}$.

\section{Isotopy Galois spaces.}

Definition 2.1. A topological space $X$ is a Galois space (representable space) provided that for each closed subset $F \subset X$ and each point $x \in X-F, F^{\prime}(x)$ contains a nondegenerate (open) subset of $X$.

Definition 2.2. A topological space $X$ is an isotopy Galois space provided that for each open subset $U$ of $X$ and each $p \in U$, there is an isotopy $H$ of $X$ with $H_{0}=I d_{X}, H_{1}(p) \neq p$ such that for each $t \in$ $[0,1], H_{t} \in(X-U)^{\prime}$.

It is evident that every isotopy Galois space is a Galois space and that $S^{1}$ and $\boldsymbol{R}$ are isotopy Galois spaces, as is every (separable metric) manifold with or without boundary of dimension greater than 1 . We omit the proof of the following proposition which is similar to the proof of the analogous result for Galois spaces [6, Proposition 3].

Proposition 2.3. A regular space $X$ is an isotopy Galois space if and only if each $x \in X$ is contained in an open set which is an isotopy Galois space.

THeOREM 2.4. Let $X$ be a completely regular space and let $Y$ be an isotopy Galois space. Then $X \times Y$ is an isotopy Galois space.

Proof. Let $(x, p) \in X \times Y$ and let $W$ be a neighborhood of $(x, p)$. There are neighborhoods $U$ and $V$ in $X$ and $Y$ respectively such that $(x, p) \in U \times V \subset W$. Let $f: X \rightarrow[0,1]$ be a mapping such that $f \mid(X-$ $U)=0$ and $f(x)=1$. Let $H$ be an isotopy of $Y$ guaranteed by Definition 2.2 for $p \in V$. Define an isotopy $G$ of $X \times Y$ by $G_{t}(a, b)=$ $\left(a, H_{t f(a)}(b)\right)$. It is a simple matter to verify that $G_{0}=I d_{X \times Y}, G_{1}(x, p) \neq$ $(x, p)$ and that for all $t \in[0,1], G_{t} \in(X \times Y-U \times V)^{\prime} \subset(X \times Y-W)^{\prime}$.

CoROLLARY 2.4. Let $\left\{X_{\alpha}: \alpha \in \Lambda\right\}$ be a collection of completely regular 
isotopy Galois spaces. Then $\times\left\{X_{\alpha}: \alpha \in \Lambda\right\}$ is a completely regular isotopy Galois space.

Example 2.5. A metrizable Galois space $X$ such that $X \times X$ is not a Galois space. Let $Q$ be the rational numbers with the usual topology. Let $\mathscr{B}_{1}=\{U \times V: U$ is open in $\boldsymbol{R}$ and $V$ is open in $Q$, and $0 \in V\}$ and let $\mathscr{B}_{2}=\{\{x\} \times W: x \in \boldsymbol{R}, W$ is open in $Q$, and $0 \notin W\}$. Let $X=\boldsymbol{R} \times Q$ with the topology generated by $\mathscr{B}_{1} \cup \mathscr{B}_{2}$.

We first show that $X$ is a Galois space. Let $(x, p) \in X$ and let $B \in \mathscr{S}_{1} \cup \mathscr{B}_{2}$ such that $(x, p) \in B$. If $p \neq 0$, then we may assume without loss of generality that $B \in \mathscr{B}_{2}$. Since $Q$ is a Galois space, there exists $h \in H(X)$ supported on $B$ such that $h(x, p) \neq(x, p)$. If $p=0$, we may assume without loss of generality that there is an open set $U$ of $\boldsymbol{R}$ and an open and closed set $V$ of $Q$ such that $B=$ $U \times V$. Since $\boldsymbol{R}$ is a Galois space, there exists $f \in H(\boldsymbol{R})$ supported on $U$ such that $f(x) \neq x$. Define $h: X \rightarrow X$ by $h(y, q)=(f(y), q)$ if $(y, q) \in B$, and $h(y, q)=(y, q)$ if $(y, q) \notin B$. Then $h \in H(X), h$ is supported on $B$ and $h(x, p) \neq(x, p)$.

Suppose that $X \times X$ is a Galois space. Let $x, y \in \boldsymbol{R}$ and let $u=$ $(x, 0)$ and $v=(y, 0)$. Let $U_{1}$ and $U_{2}$ be connected open subsets of $\boldsymbol{R}$ such that $x \in U_{1}$ and $y \in U_{2}$. Let $V$ be an open subset of $Q$ containing 0. Let $B_{1}=U_{1} \times V$ and let $B_{2}=U_{2} \times V$. There exists $h \in H(X \times$ $X)$ supported on $B_{1} \times B_{2}$ such that $h(u, v) \neq(u, v)$. Without loss of generality we may assume that $\pi_{2} h(u, v) \neq v$. Choose $w=(y, r) \in X$ such that $r \neq 0$ and such that the first component of $\pi_{2} h(u, w) \neq y$. Let $A=U_{1} \times\{0\}$. Then $h(A \times\{w\})$ is a connected subset of $X \times X$. Let $W$ be an open and closed set in $Q$ containing $r$, but not containing 0 . Then $X \times[\{y\} \times W]$ and $X \times[X-(\{y\} \times W)]$ separate $h(A \times\{w\})$ a contradiction.

The Sorgenfrey line (real line with the lower limit topology) is a completely normal homogeneous Galois space which is not metrizable. It follows from Theorem 2.4 that $\times\left\{\boldsymbol{R}_{\alpha}: \alpha \in \boldsymbol{R}\right\}$ is a completely regular homogeneous Galois space which is not normal and that $\times\left\{S_{\alpha}^{1}: \alpha \in \boldsymbol{R}\right\}$ is a normal homogeneous Galois space which is not completely normal. We omit the straightforward argument needed to establish the first of the following two examples.

EXAMPLE 2.6. A countable Hausdorff homogeneous Galois space which is not regular. Let $X$ be the points in the plane with both coordinates rational and let $\mathscr{T}$ be the topology relative to that of the deleted diameter topology of the plane. Then $(X, \mathscr{T})$ has the desired properties. 
EXAmple 2.7. A connected Galois space which is not an isotopy Galois space. Let $A=\{(x, y) \in \boldsymbol{R} \times \boldsymbol{R}: y=\sin 1 / x, 0<x\}$ and let $B=$ $\{(x, y) \in \boldsymbol{R} \times \boldsymbol{R}: x=0,-1<y<1$ and $y$ is rational $\}$. Let $X=A \cup B$ with the usual subspace topology. Let $x \in X$, let $a, c, d, e \in \boldsymbol{R}$ such that $[\{e\} \times(a, c)] \cap A=\varnothing$ and let $U=(d, e) \times(a, c)$ be an open set containing $x$. If $x \in A$, it is evident that there exists $h \in(X-U)^{\prime}$ such that $h(x) \neq x$. If $x \in B$, we may assume that $x=(0, b)$ and there is a rational number $p$ such that $a<p<b<c$.

Define $f: 0 \times[-1,1] \rightarrow 0 \times[-1,1]$ by

$$
f(0, y)= \begin{cases}\left(0, \frac{b-a}{p-a}(y-a)+a\right), & \text { if } a \leqq y \leqq p \\ \left(0, \frac{c-b}{c-p}(y-p)+b\right), & \text { if } p \leqq y \leqq c \\ (0, y) & , \text { otherwise } .\end{cases}
$$

Extend $f_{\mid B}$ to $h: X \rightarrow X$ as follows. If $y \in A \cap U$, let $h(y)=\pi^{-1} f \pi(y)$, where $\pi$ is the projection of $\boldsymbol{R} \times \boldsymbol{R}$ onto the second factor with the domain restricted to that component of $A \cap[\boldsymbol{R} \times(-1,1)]$ which contains $y$. If $y \in B$, let $h(y)=f(y)$. Then $h \in(X-U)^{\prime}$ such that $h(y) \neq y$. Thus $X$ is a Galois space. On the other hand, no point in $B$ can be moved by an isotopy, since an isotopy leaves path components invariant. Thus $X$ is not an isotopy Galois space.

We observe that every topological vector space is an isotopy Galois space. The proof of this result may be taken directly from the proof of Theorem 3.1 of [5].

3. Minimal sets and orbit attraction spaces. In this section we make use of the following notions from topological dynamics. Let $X$ be a locally compact metric space. By a flow on $X$, we mean a continuous map $f: X \times \boldsymbol{R} \rightarrow X$ such that

(i) $f(x, 0)=x$ for each $x \in X$, and

(ii) $f\left(f\left(x, t_{1}\right) t_{2}\right)=f\left(x, t_{1}+t_{2}\right)$ for all $x \in X$ and all $t_{1}, t_{2} \in \boldsymbol{R}$.

When there is no danger of confusion, we write $x t$ in room of $f(x, t)$, and if $S \subset \boldsymbol{R}$ and $A \subset X$ we write $A S$ in room of $\{a s: a \in A$ and $s \in S\}$. For $x \in X, x \boldsymbol{R}$ is called the orbit of $x ; x$ is called a fixed point if $x \boldsymbol{R}=x$, and $x$ is called a periodic point if $x$ is not a fixed point but $x t=x$ for some $t>0$. The smallest such $t$ is called the period of $x$. If $x$ is a periodic point, it is easy to see that $x \boldsymbol{R}$ is homeomorphic to $S^{1}$. A subset $A \subset X$ is invariant provided that $A \boldsymbol{R}=$ $A$, and $A$ is a minimal set provided that $A$ is closed nonempty and invariant and is minimal with respect to these properties. Evidently the orbit of every point in a minimal set $A$ is dense in $A$. We will need the following theorem which follows from results of [2]. 
Theorem 3.1. Suppose that $x \in X$, that $x$ is not a fixed point and that $t$ is a positive number restricted only by $t<\omega / 4$ in the event that $x$ is a periodic point with period $\omega$. Then there is an open subset $U$ and a set $S$ with $x \in S \subset U$ such that $U$ is homeomorphic to $S \times$ $(-t, t)$ under the mapping $(x, t) \rightarrow x t$.

DEFINITION 3.2. The topological space $X$ is an orbit attraction space provided that for each closed set $F \subset X$ and each $x \in X-F$, $F \cap \overline{F^{\prime \prime}(x)} \neq \varnothing$.

THEOREM 3.3. Every representable continuum is a strongly 2homogeneous orbit attraction space, and every orbit attraction space is a connected near homogeneous Galois space.

Proof. Let $X$ be a representable continuum, let $F \subset X$ be a closed set and let $p \in X-F$. Let $F^{\prime}$ have the discrete topology and let $\pi: F^{\prime} \times X \rightarrow X$ be defined by $\pi(h, x)=h(x)$. Then $\pi$ defines an action of $F^{\prime}$ on the compact space $X$ and it follows that $\overline{F^{\prime \prime}(p)}$ contains a minimal set $A$. There is $z \in A$ such that $A=\overline{F^{\prime \prime}(z)}$. If $z \notin F$, then $\overline{F^{\prime}(z)}$ is open so that $\overline{F^{\prime \prime}(z)}=X$. Thus $A \cap F \neq \varnothing$ and it follows that $X$ is an orbit attraction space.

Let $x, y, p, q \in X$. Since $X$ is a connected representable space, $X$ is homogeneous [4, Theorem 2]. Thus $p$ is a noncut point of $X$ and there is $h \in H(X)$ such that $h(x)=p$. Since $\left\{p^{\prime}(x): x \in X-\{p\}\right\}$ is a decomposition of the connected set $X-\{p\}$ into disjoint open sets, for $x \neq p, p^{\prime}(x)=X-\{p\}$. Thus there is $g \in p^{\prime}$ such that $g(h(y))=q$, and so $g \circ h$ is the homeomorphism needed to establish that $X$ is strongly 2-homogeneous.

It is evident that every orbit attraction space is a connected near homogeneous Galois space.

COROLlaRY 3.4. Every representable plane continuum is homeomorphic to $S^{1}$ [3, Theorem 10].

An argument similar to that of Theorem 2.4 gives the following lemma.

LEMmA 3.5. Suppose that $X$ is completely regular, that $(a, b)$ is an open interval, and that the following are given.

(1) $p \in X$ and $U$ a neighborhood of $p$,

(2) $s, t \in(a, b)$ with $s<t$, and

(3) $\eta>0$ such that $(s-\eta, t+\eta) \subset(a, b)$.

Then there is a homeomorphism $h: X \times(a, b) \rightarrow X \times(a, b)$ such that

(a) $h(p, s)=(p, t)$ and 
(b) $h \in(X \times(a, b)-U \times(s-\eta, t+\eta))^{\prime}$

THEOREM 3.6. Every nondegenerate minimal set in a flow on a locally compact metric space is an orbit attraction space.

Proof. Suppose that $X$ is such a minimal set. Let $d$ be the metric on $X$ guaranteed by the hypothesis of the theorem. Since $S^{1}$ is an orbit attraction space, we may assume without loss of generality that $X$ contains no periodic points. Let $F \subset X$ be a closed set and let $p \in X-F$. Let $W$ be a neighborhood of $F$. Since $\overline{p \boldsymbol{R}}=X$, there exist $\tau>0$ and $\eta$ with $-\tau<\eta<0$ such that $p \tau \in W$, but $p[\eta, \tau] \cap$ $F=\varnothing$. By Theorem 3.1, there exist an open set $U$ and a set $S$ with $S \subset U$ such that $p \in S$ and such that $U=S(-\tau, \tau)$ is homeomorphic to $S \times(-\tau, \tau)$. Let $\alpha=d(p[\eta, \tau], F)$. Let $\delta>0$ such that if $q \in$ $[0, \tau]$, then $\operatorname{diam}\left[S_{\delta}(p) q\right]<\alpha / 2$ where $U^{*}=S^{*}(-\tau, \tau)$ is an open set which is homeomorphic to $S^{*} \times(-\tau, \tau)$, and $S^{*}[0, \tau) \cap W \neq \varnothing$ while $S^{*}[\eta, \tau) \cap F=\varnothing$. We apply Lemma 3.5 to $U^{*}$ in order to move $p$ into $W$ with a homeomorphism which is supported on $S^{*}(\eta, \tau)$ and the theorem is proved.

The authors have been unable to find an example of an orbit attraction space or a Galois continuum which is not an isotopy Galois space. Since the pseudo-arc does not contain an arc, it is clear that this space is not an isotopy Galois space; consequently it is a natural candidate for one of the above examples. In any case, it would be interesting to know which, if any, of the properties discussed in this paper is possessed by the pseudo-arc.

Since every closed set in a metric space which admits a fixed point free flow is the invariant set of some one-dimensional flow [1, Corollary to Theorem 1], it follows that any space satisfying the hypothesis of Theorem 3.6 is an isotopy Galois space. We are in a position to establish this result directly.

THEOREM 3.7. Let $X$ be a locally compact metric space and suppose that $X$ admits a flow without fixed points. Then $X$ is an isotopy Galois space.

Proof. By Theorem 3.1, each point of $X$ has a neighborhood homeomorphic to the product of a metric space with the isotopy Galois space $(0,1)$. The result follows from Theorem 2.4 and Proposition 2.3.

ExAMPLE 3.7. A homogeneous isotopy Galois continuum which is an orbit attraction space but which is not representable. In view of Theorems 3.6 and 3.7 and [5, Theorem 4.4], it is evident that the solenoid has the requisite properties.

It has been pointed out to the authors by Professor W. Gottschalk 
(private communication) that the standard construction for manufacturing continuous flows out of discrete flows when applied to an example of a nonhomogeneous minimal set under a discrete flow due to F. B. Jones [9, Page 139] yields a non-homogeneous minimal set under a continuous flow which is a metric continuum. Thus, although every representable continuum is strongly 2-homogeneous, it follows from Theorems 3.6 and 3.7 that the minimal set constructed from F. B. Jones's example is an example of a nonhomogeneous orbit attraction space which is an isotopy Galois metric continuum.

4. Topological groups. In light of Theorem 2.4, the results of this section may be considered as immediate consequences of a rather powerful result of V. M. Gluskov which we recall here.

THEOREM 4.1 [8, Theorem A]. For every locally compact group G and every neighborhood $U$ of its identity one can find an open neighborhood $V$ of the identity which is contained in $U$ and which splits into the direct product of a connected local Lie group, L and a compact group. Moreover, if $G$ is not totally disconnected, then the neighborhood $U$ can be chosen such that in every decomposition of this form the local Lie group $L$ has positive dimension.

COROLLARY 4.2 Every locally compact topological group which is not totally disconnected is an isotopy Galois space.

COROLLARY 4.3. Every non-discrete locally compact topological group is a Galois space.

Proof. Every totally disconnected locally compact topological group is a homogeneous 0-dimensional space. Such spaces are known to be strongly locally homogeneous [7, Theorem 4.2].

\section{REFERENCES}

1. A. Beck, On invariant sets, Annals of Math., 67 (1957), 99-103.

2. N. P. Bhatia and G. P. Szego, Dynamical systems: stability theory and applications, Lecture Notes \#34, Springer Verlag (1967).

3. C. E. Burgess, Some theorems on n-homogeneous continua, Proc. Amer. Math. Soc., 5 (1954), 136-143.

4. P. Fletcher, Note on quasi-uniform spaces and representable spaces, Colloq. Math., 23 (1971), 263-265.

5. P. Fletcher and R. A. McCoy, Galois spaces, representable spaces and strongly locally homogeneous spaces, Fund. Math., 73 (1971), 85-91.

6. P. Fletcher and R. L. Snider, Topological Galois spaces, Fund. Math., 68 (1970), 143-148.

7. L. R. Ford, Jr., Homeomorphism groups and coset spaces, Trans. Amer. Math. Soc., 77 (1954), 490-497. 
8. V. M. Gluskov, The structure of locally compact groups and Hilbert's fifth problem, Amer. Math. Soc. Transl., (2) 15 (1960), 55-93.

9. W. Gottschalk and G. Hedlund, Topological Dynamics, Vol. XXXVI, Amer. Math. Soc. Coll. Pub., (1955), Providence.

Received November 18, 1971 and in revised form July 12, 1972.

OKLahoma State University

AND

Virginia Polytechnic Institute and State University 


\section{PACIFIC JOURNAL OF MATHEMATICS}

\section{EDITORS}

\author{
H. SAMELson \\ Stanford University \\ Stanford, California 94305 \\ C. R. HоввY \\ University of Washington \\ Seattle, Washington 98105
}

J. DugundJI

Department of Mathematics

University of Southern California

Los Angeles, California 90007

RICHARD ARENS

University of California

Los Angeles, California 90024

\section{ASSOCIATE EDITORS}
E. F. BeCKENBACH
B. H. NEUMANN
F. WOLF
K. YoshidA

\section{SUPPORTING INSTITUTIONS}

UNIVERSITY OF BRITISH COLUMBIA

CALIFORNIA INSTITUTE OF TECHNOLOGY

UNIVERSITY OF CALIFORNIA

MONTANA STATE UNIVERSITY

UNIVERSITY OF NEVADA

NEW MEXICO STATE UNIVERSITY

OREGON STATE UNIVERSITY

UNIVERSITY OF OREGON

OSAKA UNIVERSITY

\author{
UNIVERSITY OF SOUTHERN CALIFORNIA \\ STANFORD UNIVERSITY \\ UNIVERSITY OF TOKYO \\ UNIVERSITY OF UTAH \\ WASHINGTON STATE UNIVERSITY \\ UNIVERSITY OF WASHINGTON

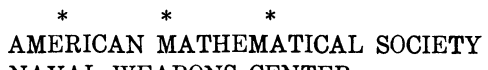 \\ NAVAL WEAPONS CENTER
}

The Supporting Institutions listed above contribute to the cost of publication of this Journal, but they are not owners or publishers and have no responsibility for its content or policies.

Mathematical papers intended for publication in the Pacific Journal of Mathematics should be in typed form or offset-reproduced, (not dittoed), double spaced with large margins. Underline Greek letters in red, German in green, and script in blue. The first paragraph or two must be capable of being used separately as a synopsis of the entire paper. The editorial "we" must not be used in the synopsis, and items of the bibliography should not be cited there unless absolutely necessary, in which case they must be identified by author and Journal, rather than by item number. Manuscripts, in duplicate if possible, may be sent to any one of the four editors. Please classify according to the scheme of Math. Rev. Index to Vol. 39. All other communications to the editors should be addressed to the managing editor, Richard Arens, University of California, Los Angeles, California, 90024.

50 reprints are provided free for each article; additional copies may be obtained at cost in multiples of 50 .

The Pacific Journal of Mathematics is issued monthly as of January 1966. Regular subscription rate: $\$ 48.00$ a year (6 Vols., 12 issues). Special rate: $\$ 24.00$ a year to individual members of supporting institutions.

Subscriptions, orders for back numbers, and changes of address should be sent to Pacific Journal of Mathematics, 103 Highland Boulevard, Berkeley, California, 94708.

PUBLISHED BY PACIFIC JOURNAL OF MATHEMATICS, A NON-PROFIT CORPORATION

Printed at Kokusai Bunken Insatsusha (International Academic Printing Co., Ltd.), 270, 3-chome Totsuka-cho, Shinjuku-ku, Tokyo 160, Japan. 


\section{Pacific Journal of Mathematics}

\section{Vol. 45, No. $2 \quad$ October, 1973}

Kenneth Paul Baclawski and Kenneth Kapp, Induced topologies for quasigroups and loops ............................................. 393

D. G. Bourgin, Fixed point and $\min -\max$ theorems $\ldots \ldots \ldots \ldots \ldots \ldots \ldots$

J. L. Brenner, Zolotarev's theorem on the Legendre symbol ............... 413

Jospeh Atkins Childress, Jr., Restricting isotopies of spheres .............. 415

John Edward Coury, Some results on lacunary Walsh series ................ 419

James B. Derr and N. P. Mukherjee, Generalized Sylow tower groups. II . . . . . . 427

Paul Frazier Duvall, Jr., Peter Fletcher and Robert Allen McCoy, Isotopy Galois

spaces .......................................... 435

Mary Rodriguez Embry, Strictly cyclic operator algebras on a Banach space ... 443

Abi (Abiadbollah) Fattahi, On generalizations of Sylow tower groups ......... 453

Burton I. Fein and Murray M. Schacher, Maximal subfields of tensor products . . 479

Ervin Fried and J. Sichler, Homomorphisms of commutative rings with unit

element .......................................... 485

Kenneth R. Goodearl, Essential products of nonsingular rings ............. 493

George Grätzer, Bjarni Jónsson and H. Lakser, The amalgamation property in

equational classes of modular lattices ...........................

507

$\mathrm{H}$. Groemer, On some mean values associated with a randomly selected simplex

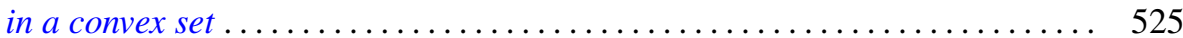

Marcel Herzog, Central 2-Sylow intersections .................... 535

Joel Saul Hillel, On the number of type-k translation-invariant groups ........ 539

Ronald Brian Kirk, A note on the Mackey topology for $\left(C^{b}(X)^{*}, C^{b}(X)\right) \ldots \ldots .543$

J. W. Lea, The peripherality of irreducible elements of lattice.............. 555

John Stewart Locker, Self-adjointness for multi-point differential operators ..... 561

Robert Patrick Martineau, Splitting of group representations ............... 571

Robert Massagli, On a new radical in a topological ring ................. 577

James Murdoch McPherson, Wild arcs in three-space. I. Families of Fox-Artin

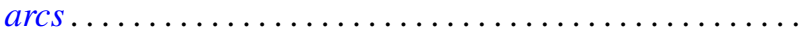

585

James Murdoch McPherson, Wild arcs in three-space. III. An invariant of

oriented local type for exceptional arcs . . . . . . . . . . . . ............ 599

Fred Richman, The constructive theory of countable abelian p-groups ........ 621

Edward Barry Saff and J. L. Walsh, On the convergence of rational functions

which interpolate in the roots of unity ..................

Harold Eugene Schlais, Non-aposyndesis and non-hereditary

decomposability..................................... 643

Mark Lawrence Teply, A class of divisible modules................... 653

Edward Joseph Tully, Jr., H-commutative semigroups in which each

homomorphism is uniquely determined by its kernel ................. 669

Garth William Warner, Jr., Zeta functions on the real general linear group ...... 681

Keith Yale, Cocyles with range $\{ \pm 1\} \ldots \ldots \ldots \ldots \ldots \ldots \ldots \ldots \ldots \ldots \ldots \ldots \ldots . \ldots \ldots$

Chi-Lin Yen, On the rest points of a nonlinear nonexpansive semigroup ........ 699 